\title{
Compte rendu de Sepik Ramu Art de Kevin Conru
}

\author{
Nicolas Garnier
}

\section{(2) OpenEdition}

Journals

Édition électronique

URL : https://journals.openedition.org/jso/11646

DOI : $10.4000 /$ jso. 11646

ISSN : $1760-7256$

\section{Éditeur}

Société des océanistes

\section{Édition imprimée}

Date de publication : 8 juin 2020

Pagination : 107-109

ISBN : 9782854301380

ISSN : 0300-953x

\section{Référence électronique}

Nicolas Garnier, "Compte rendu de Sepik Ramu Art de Kevin Conru », Journal de la Société des Océanistes [En ligne], 150 | 2020, mis en ligne le 08 juin 2020, consulté le 08 janvier 2022. URL : http:// journals.openedition.org/jso/11646; DOI : https://doi.org/10.4000/jso.11646

Ce document a été généré automatiquement le 8 janvier 2022.

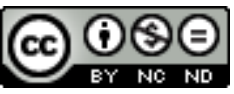

Journal de la société des océanistes est mis à disposition selon les termes de la Licence Creative Commons Attribution - Pas d'Utilisation Commerciale - Pas de Modification 4.0 International. 


\title{
Compte rendu de Sepik Ramu Art de Kevin Conru
}

\author{
Nicolas Garnier
}

\section{RÉFÉRENCE}

CONRU Kevin (éd), 2019. Sepik Ramu Art, avec des photographies de Hugues Dubois, Bruxelles, Conru Éditions, 296 p., 275 ill., graphiques et cartes.

1 Pour toute personne s'intéressant à la Nouvelle-Guinée, un nouveau livre sur le Sepik est toujours le bienvenu. Cet ouvrage, coordonné par Kevin Conru, fait suite à une série de publications récentes consacrées à cette région. On peut citer le catalogue (Peltier et al., 2016) de l'exposition itinérante Sepik (Berlin, Zurich et Paris) de 2015-2016 organisée presque simultanément à Canberra par Crispin Howarth (2015) -, le numéro spécial du Journal de la Société des Océanistes 146 faisant suite à l'exposition européenne, l'ouvrage de Markus Schindlbeck consacré au journal de bord d'Adolf Roesicke, compagnon de la première expédition scientifique menée sur le Sepik (2015) et, enfin, la traduction en anglais du rapport d'expédition d'Otto Reche en 1913 sur le Sepik (2015).

2 Cette publication fait immanquablement réfléchir à ce que recouvre le terme Sepik et à sa pertinence aujourd'hui. En effet, et ce point avait été central dans de nombreux articles d'un ouvrage pionnier, le fameux Sepik Heritage coordonné par Nancy Lukthehaus (1990), le mot Sepik recouvre des réalités géographiques assez variables. Parle-t-on d'un fleuve et de ses habitants les plus directs? Cette acception est rarement retenue, sauf peut-être dans l'ouvrage d'Otto Reche. Parle-t-on d'une région plus vaste? Alors, quelles sont les limites posées et quelle est la justification donnée à l'incorporation de populations aux cultures particulièrement diverses (et donc différentes de celles du fleuve)? Reconnaissons que, si l'ampleur de la région pose question et soulève interrogations et critiques dans Sepik Heritage, malheureusement, ni les essais du livre de Conru, ni les objets dont il rassemble des photos ne posent cette 
question. Or, les habitants du Sepik donnent aujourd'hui une définition assez limitative de cette zone géographique. Elle recoupe les divisions administratives, telles qu'elles ont été définies par le droit depuis l'indépendance du pays. On pourrait certes objecter qu'il n'est pas toujours justifié de limiter l'ambition d'un essai aux frontières administratives contemporaines. Pourtant, ce cadre administratif se construit autour d'une structure politique particulièrement vivante et active (différents niveaux électoraux, réseaux de services sociaux, groupes religieux, réseaux économiques, routes de marchés, système de transports privés, compétitions sportives...) et lui donne une véritable substance sociale.

3 L'ouvrage réunit environ deux cents œuvres qui semblent appartenir à Kevin Cornu ainsi qu'à deux familles de collectionneurs, les van Bussel et les Vanderstraete. L'ouvrage n'est pas particulièrement clair à ce propos et rien ne permet d'identifier la localisation actuelle des œuvres reproduites. Le coordinateur propose une courte introduction sur les cultures du Sepik, l'histoire coloniale de la région et des principaux collecteurs d'objets qui s'y sont rendus dans la première moitié du $\mathrm{xx}^{\mathrm{e}}$ siècle.

La contribution de Virginia-Lee Webb apporte une synthèse très appréciable des premiers travaux photographiques effectués sur le Sepik. Elle complète la publication de Markus Schindlbeck déjà citée et l'ouvrage de Brigitta Hauser-Schäublin (1989) qui réunissait déjà un nombre considérable de documents photographiques anciens et qui, grâce à eux, tentait de dresser la cartographie stylistique si complexe des styles architecturaux de la région. Elle y publiait déjà un nombre considérable de photographies du père Franz Kirschbaum. Ainsi, si on retrouve en outre des clichés déjà bien connus d'Adolf Roesicke ou d'Alfred B. Lewis (déjà partiellement publiés par Robert Welsh avec son édition critique de ses carnets de terrain en 1998), on y découvre en revanche les étonnantes photographies colorisées de Sidney Curliff ou de Karl P. Schmidt ou celle du navigateur William A. Robinson qui effectua un voyage sur le cours moyen du Sepik à l'été 1930 . Ce texte donne le détail des villages visités par ces différents photographes, ce qui permettra aux futurs chercheurs de préciser certaines localisations.

5 La contribution due à Crispin Howarth embrasse une aire culturelle comprise entre les villages situés autour des lacs Murik et la région de Bogia, non loin de l'embouchure du Ramu. L'auteur y évoque les traits communs à cette région, traits stylistiques qui se déploient par ailleurs sur une zone encore plus vaste qui s'étend largement jusqu'à Wewak et comprend les îles Schouten. Cette aire stylistique n'est pas le résultat d'un mode de classification exogène et est parfaitement revendiquée localement. Je me souviens qu'en 2000, des habitants d'une zone allant de l'île de Kairiru jusqu'au village de Mendam à Murik affirmaient que leur style était celui des "petits couteaux" (remarquable par la finesse du décor gravé sur les volumes). Ils l'opposaient à celui du cours moyen du Sepik, qu'ils appelaient le style du "grand couteau». Selon les premiers, les habitants du moyen Sepik produisaient des sculptures qui se distinguaient des leurs par l'absence d'un fin décor et par la robustesse des volumes sculptés. Dans son article, Crispin Howarth insiste non seulement sur la proximité stylistique qui rapproche les figures et les masques sculptés de la région du Sepik et de l'embouchure du Ramu, mais aussi sur les échanges nombreux auxquels se livrent ces villages, y compris d'œuvres sculptées, dont en particulier d'importants tambours à fente. Il met également en avant un concept-clé, gai'in, qu'il traduit par corps-pirogue, concept qui permet de comprendre la manière dont les objets sculptés deviennent les réceptacles 
d'êtres surnaturels (ancêtres ou esprits). Ce n'est pas sans évoquer la lecture qu'avait proposée Brigitta Häuser-Schaublin des masques mai chez les Iatmuls (1976-77 et 2017). Ce qui lie une figure sculptée à l'être qu'elle est supposée incarner est une problématique cruciale dans la statuaire du Sepik et, d'une manière beaucoup plus large, de la statuaire en général ${ }^{1}$. Car ce qu'approche brièvement Crispin Howart en citant l'article de Matthew Tamaone de 1977 pourrait être programmatique de futures recherches sur la figuration dans cette région mélanésienne (p. 53). En effet, le masque ou la statue ne peut être considérée comme la partie matérielle d'un être que la partie immatérielle viendrait habiter. Une dichotomie matérielle/spirituelle n'existe pas dans la région (ou plutôt, on n'en connaît pas d'exemple), mais il existe en revanche des configurations particulièrement complexes. Incidemment, Crispin Howarth avec sa référence sur les corps-pirogues (body-canoe), nous propose une voie interprétative nouvelle. Je ne pense pas qu'il faille voir dans la pirogue le mode de transport ou l'enveloppe matérielle qui offrirait à la partie immatérielle d'être le refuge. La pirogue, tout comme la notion de "corps » (dans son acception quotidienne), ne désignent ni la personne ni le corps biologique, mais, dans les deux cas, ce qui relie la personne, comme individu, à un collectif. De la même manière, la pirogue peut désigner, comme à Chambri par exemple, à la fois un instrument de locomotion et l'ensemble des frères classificatoires. Dans ce sens le terme $k e$ fait se superposer l'idée de pirogue de guerre et celle de clan patrilinéaire. Si l'on revient à l'exemple de la région de Murik, et sous réserve d'une étude plus approfondie, la statuaire permettrait de lier un être particulier (ancêtre ou « esprit ») à un groupe humain plus large. La statuaire n'est plus l'hôte matériel d'un être dématérialisé, mais ce qui rend possible la socialisation d'un être dans une dynamique de groupe.

6 La dernière partie du livre est écrite par Barry Craig. Elle met en relation l'essentiel des objets publiés dans le livre et des informations ethnographiques. Barry Craig suit un parcours géographique qui l'entraîne à travers l'aire iatmul, chez les Kwoma et les Abelam. Il descend ensuite le fleuve pour rejoindre le Blackwater et le Karawari, puis la rivière Yuat, le Keram, la région des lacs Murik et, enfin, les parties basses et médianes du Ramu. Il porte son attention à certains éléments, par exemple aux travaux de vannerie et aux boucliers. La construction de cette partie rappelle la manière dont avait été conçu le catalogue des chefs-d'œuvre du musée national de Port Moresby (Craig 2010). S'il est assez frustrant de ne lire que quelques pages sur chacune des cultures, la grande érudition et l'expérience du terrain de l'auteur rendent passionnante la lecture de ce long chapitre.

7 En 1988, le PNG National Museum publiait un remarquable petit opuscule, portant presque le titre de la publication de Kevin Conru. Il était coordonné par Pamela Swadling, Brigitta Hauser-Schäublin, Paul Gorecki et Frank Tiesler et le texte concis mais précis était accompagné de très riches illustrations évoquant l'archéologie, la culture matérielle et l'environnement de cette région. L'ouvrage avait pour ambition de s'adresser à un public très général, aux spécialistes de la région, sans doute aux amateurs d'art, mais surtout aux habitants de la Papouasie Nouvelle-Guinée. On se prend parfois à regretter ce type de publications socialement plus transversales et qui trouvaient alors facilement leur chemin jusque dans de nombreuses maisons du Sepik et du Ramu. À l'avenir, il faudrait que nous, qui travaillons sur la Papouasie NouvelleGuinée, puissions imaginer un financement de nos publications qui permette de les faire partager plus largement. 


\section{BIBLIOGRAPHIE}

CRAIG Barry (ed.), 2010. Living Spirits with Fixed Abodes : The Masterpieces Exhibition, Papua New Guinea National Museum and Art Gallery, Adelaide, Crawford House Publishing.

HAUSER-SCHÄUBLIN Brigitta, 1976-1977. Mai-Masken der Iatmul, Papua New Guinea, Stil, Schnitzvorgang, Auftritt und Funktion, Verhandlungen der Naturforschenden Gesellschaft 87-88 (Basel, Switzerland), pp. 119-145.

HAUSER-SCHÄUBLIN Brigitta, 1989. Kulthäuser in Nordneuguinea, Berlin, Akademie-Verlag. HAUSER-SCHÄUBLIN Brigitta, 2017. The Dancers Who Became Transformed into Wood : the mai masks of the Iatmul, Papua New Guinea, Oceania 87, 3, pp. 234-260.

HOWARTH Crispin (ed.), 2015. Myth + Magic : Art of the Sepik River, Papua New Guinea, Canberra, National Gallery of Australia.

KAUfMANn Christian, Philippe PelTier \& Markus SCHINDLBECK (éds), 2018. Journal de la Société des Océanistes 146 : Le Sepik : société et production matérielle.

LUTKEHAUS Nancy et al. (eds), 1990. Sepik Heritage : Tradition and change in Papua New Guinea, Bathurst, Crawford House Publishing.

Peltier Philippe, Christian KAUfMAnn \& Markus SCHIndbeck (eds.), 2016. Sepik, Arts de PapouasieNouvelle-Guinée, Paris, Skira, Musée du quai Branly.

RECHE Otto, 1913 [2015]. The Empress Augusta/Sepik River, Dunedin, University of Otago [trad. de John Dennsion].

SCHINDLBECK Markus, 2015. Unterwegs in der Südsee : Adolf Roesicke und seine Fahrten auf dem Sepik in Neuguinea, Berlin, Nicolai \& Ethnologishes Museum, Staatlische Museen zu Berlin.

SWADLING Pamela, Brigitta HAUSER-SCHÄUBLIN, Paul GORECKI et Franck TIESLER, 1988. The Sepik - Ramu, Port Moresby, PNG National Museum.

TAMOANE Matthew, 1977. Kamoai of Darapap and the Legend of Jari, Gigibori 3 (2), p. 19.

WARBURG Aby, 2015. Les statues votives de cire, in Essais Florentins, Paris, Hazan, pp. 124-127.

WELSCH Robert L. (ed.), 1998. An American Anthropologist in Melanesia : A. B. Lewis and the Joseph N. Field South Pacific Expedition 1909-1913, Honolulu, University of Hawai'i Press.

\section{NOTES}

1. On pourrait voir dans les notes consacrées par Warburg aux voti, ces figures votives de cire des églises florentines à la Renaissance, un intérêt précoce qui témoigne d'une fertilisation croisée de l'anthropologie et de l'histoire de l'art (Warburg, 2015). 


\section{AUTEUR}

NICOLAS GARNIER

Paris, MQB-Jacques Chirac 